\title{
Technologies and Operations for High Voltage Corona Detection with UAVs
}

\author{
Andrew J. Moore \\ NASA Langley Research Center \\ Hampton, VA, USA
}

\author{
Matthew Schubert \\ Analytical Mechanics Associates, Inc. \\ Hampton, VA, USA
}

\author{
Nicholas Rymer \\ National Institute of Aerospace \\ Hampton, VA, USA
}

\begin{abstract}
Autonomous UAV transmission line inspection served as a reference mission for NASA demonstration of UAV deployment for economic benefit; this paper reports corona sensing advances in development of that reference mission. Unmanned aerial vehicles can serve as a platform for autonomous sensing and location of high voltage coronal discharge. Simple processing of commercial corona camera imagery can automate discharge localization and documentation. Inexpensive ultraviolet point sensors can sense discharge when carried close to the defect with a UAV. Augmented with a parabolic mirror, point sensor range can be increased to a safe inspection standoff distance, at the cost of a narrowed field of view. Results from a test flight of an augmented UV sensor are described. The imaging approach is superior in sensitivity and acquisition time, while the point sensor approach has superior size, weight, cost and durability advantages.
\end{abstract}

Index Terms - corona detection, inspection, UAV, ultraviolet.

\section{INTRODUCTION}

Safe operations of small UAS vehicles in uncontrolled airspace is a major goal of NASA's Unmanned Aerial System (UAS) Traffic Management (UTM) project [1]. A far-term aim of UTM research and development is to accommodate small UAS operations throughout the National Airspace System. Research on UAS autonomy and navigation at low altitudes and beyond visual line-of-sight operations is a particularly emphasized in the UTM program.

To advance unmanned aerial vehicle (UAV) navigation technology while accomplishing a task with economic and societal benefit, a high voltage electrical infrastructure inspection reference mission was designed. An integrated airground platform was developed for this mission and tested in experimental flights [2][3]. The airborne component of the platform was a multirotor UAV built from commercial off-theshelf hardware and software, and the ground component was a commercial laptop running open source software. While the platform design has much in common with other ground station and multirotor aircraft combinations in current use, the autonomy and navigation requirements of this reference mission are exceptionally demanding and a successful execution would represent an advance in UAS aviation. Technological and operational advances in pursuit of successful execution of this reference mission may inform private sector development of autonomous high voltage infrastructure by UAVs.

In electrical power transmission, great care is taken to avoid sharp protrusions on structures that are on or near high voltage conductors, to avoid a local concentration of the electric field that exceeds the threshold for coronal discharge. Electron avalanches from high-potential points produce an ultraviolet (UV) photon spray via impact ionization and subsequent recombination of atmospheric plasma. While most coronas are benign, some are indicative of severe degradation that requires immediate attention; location, diagnosis and disposition of coronas is a necessary component of transmission line inspection. However, inspection is infrequent due to the cost and difficulty of UV-band image capture and due to the cost of human inspection of UV-band imagery. More frequent inspection of power transmission infrastructure may be possible by low-cost aerial (UAV) sensing and automated analysis.

Corona sensing using a UAV was selected as a focus goal of this UTM reference mission. Two UV sensing methods were studied: image-based sensing using a commercial corona camera [4][5] and point sensing using a commercial line of UV detectors [6] that use the photoelectric effect to count narrowband UV photons in a Geiger-Mueller tube and circuit configuration. Experimental results and the advantages and disadvantages of each of these methods in airborne corona sensing is discussed in this paper. We find that both methods are viable for UAV-based corona detection, and that each method has comparative benefits and drawbacks.

Line crawling robots have been developed and improved over the last 30 years to carry out some autonomous high voltage infrastructure inspection tasks (see for example [7][8][9] and [10][11] for reviews). There has not been a

This work was performed with support from the NASA Safe Autonomous Systems Operations and Unmanned Aerial System Traffic Management programs and under space act agreements with Dominion Resources and Southern Company. 
thorough study of the relative advantages of robotic crawlers versus UAVs for various autonomous high voltage infrastructure inspection. However, this work and other demonstrations of UAVs in this domain [12][13][14] suggest that such a comparison is worthwhile. The conclusions in this work regarding the suitability of these two corona detection methods for UAVs generally apply to their use on crawling robot inspection systems.

\section{CORONA CAMERA METHOD}

The dielectric response to a high E field is a complex, multiregime phenomenon [15][16], particularly when the field arises from an alternating (e.g., $60 \mathrm{~Hz}$ ) source: cathodal and anodal coronas initiate, propagate and extinguish in each positive and negative half-cycle of alternation [17]. The density of coronal ionization and recombination sites decreases monotonically with distance from the initiation point; in a planar projection into the viewport of a UV imager, the photon distribution appears as a solid ball of emission in the nucleus and a speckled halo in the periphery [18][19].

Compared to point sensors, corona cameras are superior in sensitivity and speed of acquisition. They are exquisitely sensitive to the weak UV photon flux density produced by coronas, and a user with limited training can spot a corona from the ground at a distance of 300 feet or more. Their field of view is wide enough to capture UV emission in an area containing several mid-span conductors or a group of insulators and conductor attachments at a transmission line tower.

The class of UAVs widely known as sUAS (small unmanned aerial systems) that weigh $25 \mathrm{~kg}$ or less, such as the ones employed in this flight series, are subject to far fewer regulations than their heavier counterparts. However, sUAS are can only carry small, light payloads. Relative to point sensors, corona cameras are less well-suited for sUAS applications due to greater size, weight, cost, and fragility. Typical UV cameras measure $20 \mathrm{~cm}$ or more in their longest dimension, and weigh more than $1 \mathrm{~kg}$. Typical rotary sUAS vehicles used for transmission line inspection cost on the order of USD \$3000 at this writing, so that loss of a vehicle is not a large financial risk. Commercial UV cameras cost an order of magnitude more, and so the risk of camera damage will be a dominant concern until sUAS autonomy has advanced to the point that this risk is lowered. Alignment of the UV-band and visible-band optical paths is a key to their effectiveness, but that alignment is sensitive to the vibration and mechanical shock that is typical of sUAS operation. For these reasons, a corona camera was not flown on an sUAS in this flight series. Instead, ground-based corona imagery was used to analyze phenomenology and to design a corona fault detection method.

In conventional use, a ground-based or helicopter-based inspector locates the site of coronal emission with a UV-band camera and then records visible imagery of the site so that expert analysts can assess whether the corona is benign or indicative of a serious problem. Both a close-up visible-band image at the corona center and a zoomed-out visible-band image of the UV emission area are typically recorded to diagnose the fault and effect repairs. The close-up image provides clues about the reason for the fault and the zoomed- out image provides context needed to assess repair approaches and possible secondary causes of the fault.

An UAV-based inspection operation could collect equivalent diagnostic imagery if the aircraft is equipped with a corona camera, an aligned visible camera, and computing hardware capable of processing the corona camera output. As shown in Figure 1, difference of box (DoB) filtering of UVband imagery can determine the existence and location of a corona site. A DoB filter value that is erratic or nonmonotonic with increasing kernel size indicates ambient noise or a corona site that is only partially within the UV camera field of view (top right of Figure 1), while a DoB filter value which increases smoothly and monotonically with filter kernel size (middle right of Figure 1) strongly indicates a positive detection within the UV camera field of view. If the filter output indicates that a corona is detected, the box filter dimensions can provide the framing of both the zoomed-in close-up and the zoomed-out context visible-band image records [20].
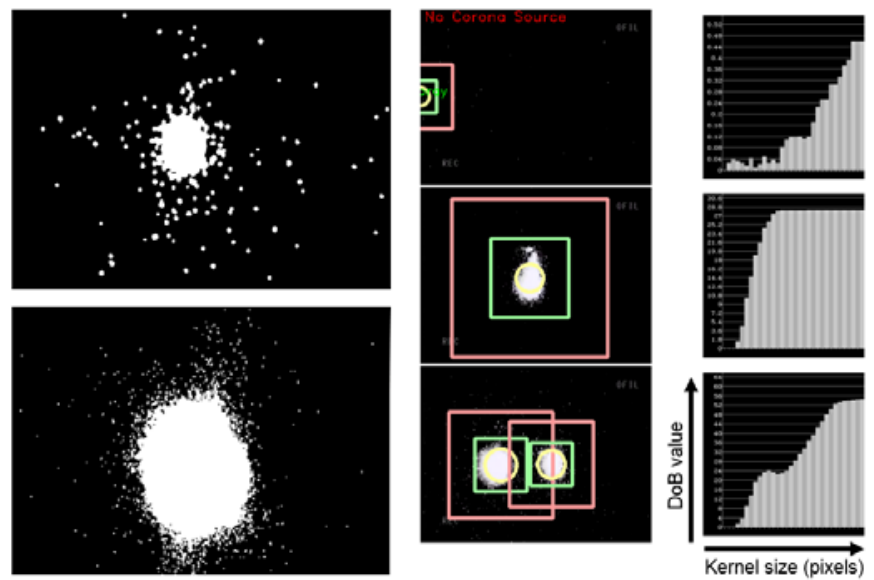

Figure 1. Left: two examples of UV corona discharge imagery captured at midrange camera gain. Right: difference-of-box (DoB) overlay of greyscale corona image (left) and scale space representation (right) with DoB value plotted as a function of kernel size. If the value is nonmonotonic and erratic (top right), the corona camera field of view is not centered on a corona figure and is imaging ambient emission or peripheral corona spray. A smooth monotonic value through scale is the signature of a well centered image of a single corona (middle right). The green box frames an ideal visible-band inspection image, and the red box frames an image window with inspection context. Well-separated coronas have a smooth but modal score through scale (not shown), while near, adjacent coronas have a nearly monotonic value though scale (bottom right).

With this or similar automated processing, an airborne UV camera can detect coronas, actively collect inspection imagery, and stamp the imagery with the UAV's GPS location. Autonomous flights by UAVs carrying corona cameras could thus inspect high voltage transmission lines and create archives of potential fault locations and fault imagery that analysts could access post-flight to make repair and maintenance decisions.

\section{POINT SENSOR METHOD}

Compact UV sensors may eventually supplement or replace corona cameras for finding potential high-voltage transmission line faults [21][22] due to their smaller size, lower cost, and greater mechanical durability. However, UV point sensors are less sensitive than corona cameras and must be swept multiple 
times across an area to collect comparable inspection data. As a result, compact UV sensors have not yet been applied broadly in transmission line inspection.

Recent technology advances may ameliorate these disadvantages. Device sensitivity has more than doubled in recent years. Prior versions of the device could sense a $100 \mathrm{kV}$ corona only within $1.2 \mathrm{~m}(4 \mathrm{ft})$, but more recent versions can do so at distances up to about $3.6 \mathrm{~m} \mathrm{(12} \mathrm{ft)} \mathrm{[2][21].} \mathrm{This} \mathrm{distance}$ can be increased further with optical augmentation (at the cost of a narrowed field of view) as described below. The ready availability of UAVs provides a means to carry the sensor close to the site of UV emission and to sweep it as needed to pinpoint the fault.

\section{A. Flight tests with unaugmented point sensor}

A compact ultraviolet sensor mounted on the UAV can in principle locate corona "hot spots" so long as the UAV flight path adequately samples the airspace near the power grid structures. Figure 2 shows a flight at Dominion Virginia Power's Chester training facility in which a UV source was mounted on a substation structure and a UAV flew at a standoff

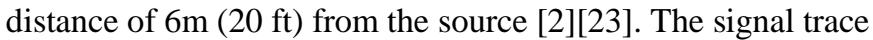
from the point sensor (top right inset of Figure 2) in the course of the flight matched the proximity to the source, and corona camera imagery (bottom right inset of Figure 2) verified the presence of UV photon flux throughout the flight. In this experiment, the corona source was set to emit UV at an intensity higher than a true corona, so that the sensor at this distance could register a signal. (See [2] for point sensor calibration details.) This experiment was repeated at a second, higher altitude structure (a switching tower) at the same $6 \mathrm{~m}(20 \mathrm{ft})$ standoff distance [2] at this facility, and at the altitude of the conductors and tower cross-arms of a $500 \mathrm{kV}$ structure at Southern Company's Klondike training facility in Lithonia, Georgia [3].

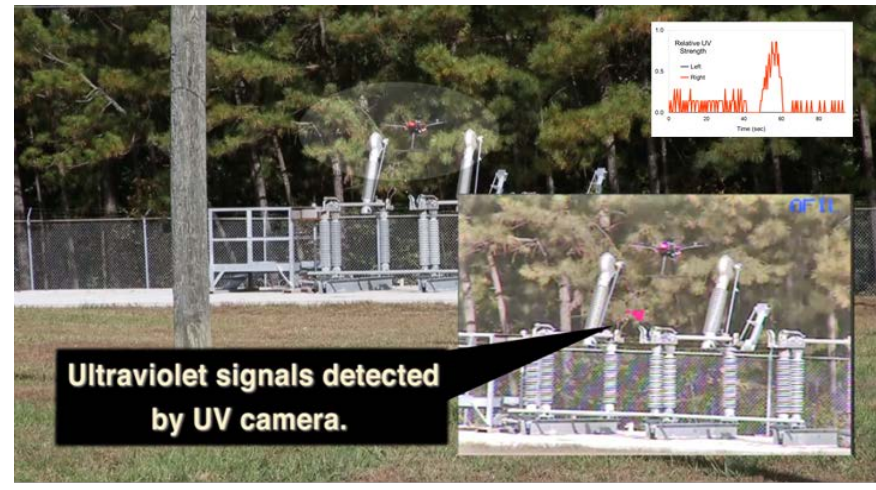

Figure 2. Validation of the compact UV sensor signal with corona camera in a substation measurement flight. The visible camera image shows the UAV above the substation structure (background; UAV is in lightened ellipse). The inset at top right shows the compact UV sensor recording, which reaches a maximum as the UAV passes the corona generator. The inset at bottom right shows the corona camera record; the bright red blobs at the center of the image are locations of UV emission.

\section{B. Flight tests with optically augmented point sensor}

While the UV point sensors tested have a wide $\left(>90^{\circ}\right)$ field of view, their detection range is not sufficient for safe corona detection in autonomous UAV inspection flights. At high altitudes and at low altitudes far from vegetation and ground structures, GPS accuracy is about two meters in latitude and longitude and approximately 2 times poorer in altitude accuracy (95\% confidence interval, [24]). However, GPS can be unreliable near reflective ground structures, and GPS-based UAV positioning is correspondingly unreliable. Without careful manual piloting or bulky, high-precision positioning equipment, inspection standoff distance must include a safety buffer for obstacle avoidance. For autonomous transmission line inspection with multi-rotor UAVs this additional buffer imposes a sensor range requirement of $6.1 \mathrm{~m}$ (20 feet) to allow time for pilot intervention as discussed in [3] [25].

To increase range to a distance that provides standoff inspection distances that mitigate collision, UV detectors were augmented with a shallow parabolic mirror to extend detection range. Sensor-dish assemblies were fabricated, tested, and flown mounted on the bottom of a UAV [25]. This novel design detected a UV signal strength equal to a $100 \mathrm{kV}$ corona at nearly twice the distance of the unaugmented sensor, though with a narrower field of view. From a range of dish diameters and curvatures, a $12.7 \mathrm{~cm}$ (5 inch) dish was selected for flight tests. Its field of view is $\pm 8^{\circ}$ at half power; at a distance of $6.1 \mathrm{~m}$ (20 feet), the half-power detection window of the augmented sensor is approximately $1.8 \mathrm{~m}$ (6 feet) in diameter. The sensor-mirror assembly mounted on a UAV is shown in Figure 3.
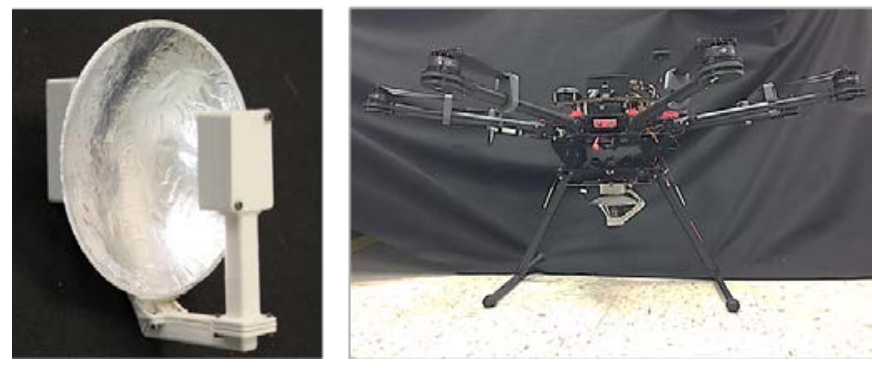

Figure 3. Sensor-mirror UV detector and vehicle mounting. A dish with $12.7 \mathrm{~cm}$ (5 inch) diameter and 0.35 focal ratio (left) provided the desired $6.1 \mathrm{~m}(20 \mathrm{ft})$ range over a $16^{\circ}$ half-power beamwidth. For mechanical stability, additional support struts and an angled sensor arm were used in the detector mounted on the UAV (right).

A corona source fastened to tripod was placed at a known location (top left of Figure 4), and a set of zigzag waypoints was created within a $7.5 \mathrm{~m} \times 7.5 \mathrm{~m}$ box centered on that location at three elevations ( $3 \mathrm{~m}, 5 \mathrm{~m}$ and $7 \mathrm{~m}$ ) above the source. UV signal strength was transmitted to the ground station as the UAV traversed these waypoints. After the flight, telemetry was corrected for UAV attitude and rendered in $\mathrm{kml}$ format. A one meter line representing UV sensor direction was constructed extending from the UAV position in the body frame of the aircraft and rotated to the earth frame using yaw, pitch, and roll intrinsic Tait-Bryan angle rotations [26]. The color of the placemark and the sensor direction line was set on a gradient scale from green to red based on the UV signal strength: green at the minimum strength of $1-2 \mathrm{~Hz}$ and red at the maximum strength of $13-14 \mathrm{~Hz}$. A signal strength of $0 \mathrm{~Hz}$ (no signal) was colored grey.

The results are shown at the bottom left (top-down view) and right (side view) in Figure 4. At a distance of 9.1 m (30 feet; out of frame), the measured UV intensity matched the 
calibrated response level for the $12.7 \mathrm{~cm}$ (5 inch) dish. Signal magnitude varied with distance and pointing angle as expected from the sensor-mirror characterization.

The field of view limitation of the augmented point sensor is evident in these results. To inspect mid-span, multiple flights (one for each conductor) are needed. To inspect a tower area, a flight path must be designed which fully samples the volume around the tower.
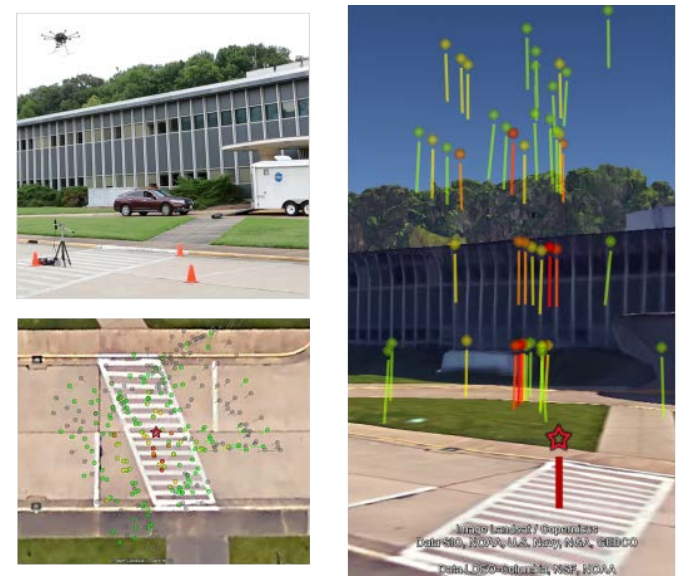

Figure 4. Flight results with UV point sensor augmented with reflective dish. Top left: video frame from measurement flight. A corona generator was placed on a tripod at $1 \mathrm{~m}$ elevation, and the UAV was flown in a zigzag pattern at elevations three, five, and seven meters higher. Bottom left: rendering of flight telemetry, top-down view. The star indicates the UV source location. Measured UV intensity is indicated by color: $0 \mathrm{~Hz}$ (grey), 1$14 \mathrm{~Hz}$ (green-to-red color scale). Right: side view. Direction lines are drawn in the mirror pointing direction. Points with low UV count $(0-2 \mathrm{~Hz})$ are removed in the view at right to reduce clutter.

\section{DISCUSSION}

Airborne avionics to sense transmission line faults and onboard computing to safely guide a UAV near high-voltage structures are essential components of an autonomous UAVbased electrical grid inspection capability [27]. A fleet of UAVs equipped with these components could autonomously examine high-voltage structures, pinpointing locations of the grid with damage and equipment malfunction that represent potential or actual risks to power delivery. If distributed across a power grid (for example, at substations), this fleet could serve as the detection foundation of a self-diagnosing power grid (Figure 5). Imagery and other telemetry from the UAV deployments could then be interpreted remotely by experienced grid operation crews and line crews, enabling the rapid dispatch of a nearby line crew to repair the fault.

Most critical high voltage faults can be diagnosed with inspection in the visible, long wave infrared, and ultraviolet bands. Due to their wide variety, faults detectable in the visible band require extensive analysis by trained personnel (see for example [28]). Faults detectable in the long wave infrared band are more specific [29] and may be possible with automated methods [30]. Faults detectable in the ultraviolet band are limited to corona emission, and are amenable to automation based on image processing [20][31] or photon counting [12][18]. UAV-borne sensors from all three bands are suitable in a self-diagnosing power grid architecture. The analysis cost of inspection data from the visible and long wave infrared bands will be high at first. The analysis cost of inspection data from the ultraviolet band is already low enough to justify deployment.

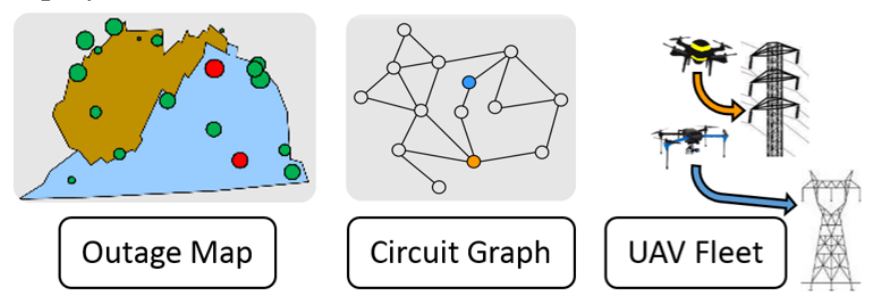

Figure 5. Components of a self-diagnosing power grid. UAVs cached at locations across the grid, such as electrical substations, can be deployed on demand to locate and characterize the faults based on geographic outage maps and topological circuit maps.

\section{CONCLUSIONS}

This report has summarized sensor evaluation and development in the course of executing a high voltage power line inspection reference mission using UAVs. A UV imaging (corona camera) approach and a point sensor approach were compared for diagnostic efficacy and for deployment readiness on a small UAV. The imaging approach is superior in sensitivity and acquisition time, and automation to process its results is readily attainable. The point sensor approach requires optical augmentation to extend its range to a safe standoff distance and requires more flight time, but has superior size, weight, cost and durability advantages. In the near term, both approaches are viable as a sensing component of a UAV-based self-diagnosing electrical grid.

\section{ACKNOWLEDGMENT}

Dominion Virginia Power and Southern Company were key partners in this research and flight campaign.

\section{REFERENCES}

[1] T. Prevot, J. Rios, P. Kopardekar, J. Robinson, M. Johnson, J. Jung, "UAS Traffic Management (UTM) Concept of operations to safely enable low altitude flight operations", 16th AIAA Aviation Technology Integration and Operations Conference AIAA Aviation (AIAA 20163292).

[2] A.J. Moore, M. Schubert, and N. Rymer. "Autonomous Inspection of Electrical Transmission Structures with Airborne UV Sensors-NASA Report on Dominion Virginia Power Flights of November 2016." NASA Technical Memo 2017-219611 (2017).

[3] A.J. Moore, et al. "UAV Inspection of Electrical Transmission Infrastructure with Path Conformance Autonomy and Lidar-based Geofences, NASA Report on UTM Reference Mission Flights at Southern Company Flights November 2016," NASA Technical Memo 2017-219673 (2017).

[4] M. Lindner, S. Elstein, P. Lindner, J. M. Topaz, A. J. Phillips, "Daylight corona discharge imager", Proc. 11 Int. Symp. on HV Eng., vol. 4, pp. 349-352, 1999.

[5] W. L. Vosloo, G. R. Stolper, P. Baker, "Daylight corona discharge observation and recording system", Proc. 10 Int. Symp. on HV Eng., vol. 6, pp. 161-164, 1997.

[6] Hamamatsu Flame Sensor UV TRON, Hamamatsu Photonics K.K., Electron Tube Center, Shimokanzo, Toyooka-village, Iwata-gun, Shizuoka-ken, 438-0193, Japan

[7] P. Debenest et al., "Expliner-Robot for inspection of transmission lines." In Robotics and Automation, 2008. ICRA 2008. IEEE International Conference on, pp. 3978-3984. IEEE, 2008. 
[8] A. Phillips et al., "Autonomous overhead transmission line inspection robot (TI) development and demonstration." In 2nd International Conference on Applied Robotics for the Power Industry (CARPI), IEEE, 2012.

[9] N. Pouliot, D. Mussard, and S. Montambault," Localization and archiving of inspection data collected on power lines using LineScout Technology." In 2nd International Conference on Applied Robotics for the Power Industry (CARPI), IEEE, 2012.

[10] R.S. Gonçalves and J.C Carvalho, "Review and latest trends in mobile robots used on power transmission lines," International Journal of Advanced Robotic Systems, vol. 10, pp. 408, 2013.

[11] O. Menendez, F. A. Auat Cheein, M. Perez and S. Kouro, "Robotics in Power Systems: Enabling a More Reliable and Safe Grid," in IEEE Industrial Electronics Magazine, vol. 11, no. 2, pp. 22-34, June 2017.

[12] B. Wang, X. Chen, Q. Wang, L. Liu, H. Zhang and B. Li, "Power line inspection with a flying robot," In 1st International Conference on Applied Robotics for the Power Industry (CARPI), 2010, pp. 1-6, 2010.

[13] T. Santos et al., "PLineD: Vision-based power lines detection for Unmanned Aerial Vehicles," In 2017 IEEE International Conference on Autonomous Robot Systems and Competitions (ICARSC), pp. 253-259. 2017

[14] C. Ippolito, K. Krishnakumar and S. Hening, "Preliminary results of powerline reconstruction from airborne LiDAR for safe autonomous low-altitude urban operations of small UAS," 2016 IEEE SENSORS, pp. 1-3. 2016

[15] Frank William Peek, Dielectric phenomena in high voltage engineering (McGraw-Hill), 1920.

[16] M. Goldman and R.S. Sigmond, “Corona and insulation." IEEE Transactions on Electrical Insulation, vol. 2, pp.90-105, 1982.

[17] A. E. W., Austen, and S. Whitehead, Discharges in Insulation Under Alternating-Current Stresses, Journal of the Institution of Electrical Engineers III, 88(1), (1941), pp. 18-22.

[18] K. M. Shong, Y. S. Kim, and S. G. Kim, Images Detection and Diagnosis of Corona Discharge on Porcelain Insulators at $22.9 \mathrm{kV} \mathrm{D} / \mathrm{L}$, IEEE Int. Symp. SDEMPED 2007, pp. 462-466

[19] L.B. Loeb (1965). Electrical coronas, their basic physical mechanisms. Berkeley, CA: Univ of California Press, 1965.

[20] M. Schubert, and A. J. Moore. "Morphological processing of ultraviolet emissions of electrical corona discharge for analysis and diagnostic use." Applied Optics 55.7 (2016): 1571-1572.
[21] N. Rymer, A. J. Moore, M. Schubert, and J. Hubbard. "Directional UV Detection for UAV Power Line Inspection.” Online video clip. YouTube, 14 September 2015. Web. 8 March 2017. https://youtu.be/GPePalYC28M

[22] M. Blanks, Mark. "UAS Applications." Introduction to Unmanned Aircraft Systems, Boca Raton, Fl: CRC Press, Taylor \& Francis Group, (2015): 19.

[23] A.J. Moore, M. Schubert, and N. Rymer. "Drone senses power line corona while tracked in U.S. airspace: NASA-Dominion UAV Flight Test with Compact Corona Sensor and UTM Tracking.” Online video clip. YouTube, 31 March 2017. Web. 31 March 2017. https://youtu.be/8shsiM-PMjs

[24] Civil Report Card On GPS Performance Nov 2016. (2016, December). Retrieved March 15, 2017, from http://www.nstb.tc.faa.gov/reports/ReportCards/2016 11.pdf

[25] A.J. Moore, N. Rymer, M. Schubert, and S. Dorsey. “A compact, lightweight method to detect high voltage corona with UAVs," unpublished, 2017.

[26] J. Diebel, "Representing attitude: Euler angles, unit quaternions, and rotation vectors.” Matrix, 58(15-16), 1-35 (2006).

[27] J. Toth and A. Gilpin-Jackson, "Smart view for a smart grid unmanned aerial vehicles for transmission lines," in 1st International Conference on Applied Robotics for the Power Industry (CARPI), 2010, pp. 1-6, 2010.

[28] X. Wang and Y. Zhang, "Insulator identification from aerial images using support vector machine with background suppression." In Unmanned Aircraft Systems (ICUAS), 2016 International Conference on, pp. 892-897. IEEE, 2016.

[29] T.L. Hurley, "Infrared qualitative and quantitative inspections for electric utilities", Proc. SPIE 1313 Thermosense XII, pp. 6-24, 1990.

[30] A. S. N. Huda, S. Taib, "Suitable feature selection for monitoring thermal condition of electrical equipment using infrared thermography", Infrared Physics \& Technology, vol. 61, pp. 184-191, 2013.

[31] C. Vidan, and M. Maracine, "Corona Discharge Classification Based on UAV Data Acquisition." In Control Systems and Computer Science (CSCS), 2017 21st International Conference on, pp. 690-695. IEEE, 2017. 